\title{
Reversible Cerebral Vasoconstriction Syndrome with Transient Splenial Lesions after Delivery
}

\author{
Akiyuki Hiraga ${ }^{1}$, Kyosuke Koide ${ }^{1}$, Yuya Aotsuka ${ }^{1}$ and Satoshi Kuwabara ${ }^{2}$
}

\begin{abstract}
Reversible cerebral vasoconstriction syndrome (RCVS) is characterized by segmental vasospasm and it is often accompanied by either posterior reversible encephalopathy syndrome or stroke. However, other MRI abnormalities have rarely been reported. A 28 -year-old woman presented with a thunderclap headache immediately after delivery; MRI showed segmental vasoconstriction and an abnormal signal in the splenium of the corpus callosum. Neuroimaging abnormalities normalized 20 days after the first examination. Only two cases of RCVS with transient splenial lesions (TSL) have so far been reported. Both cases occurred postpartum like ours, indicating that delivery may be a trigger for the development of both TSLs and RCVS.
\end{abstract}

Key words: magnetic resonance imaging, reversible cerebral vasoconstriction syndrome, transient splenial lesion, delivery, pregnancy

(Intern Med 55: 3357-3359, 2016)

(DOI: 10.2169/internalmedicine.55.7192)

\section{Introduction}

Reversible cerebral vasoconstriction syndrome (RCVS) is characterized by severe headaches, with or without other acute neurological symptoms, and by the diffuse segmental constriction of the cerebral arteries that resolves spontaneously within three months (1). In addition to the simple vasoconstriction demonstrated by magnetic resonance angiography (MRA), RCVS-associated abnormalities, including posterior reversible encephalopathy syndrome (PRES) and/or ischaemic/haemorrhagic strokes (accompanied by RCVS), have also been demonstrated by magnetic resonance imaging (MRI). However, cases of RCVS with transient splenial lesions (TSL) detected by MRI are rare; only two such cases have been reported previously $(2,3)$. We herein present a case of RCVS with TSL after delivery without eclampsia.

\section{Case Report}

A 28-year-old woman with no history of migraine or any other disease developed a thunderclap headache immediately after an uncomplicated pregnancy and spontaneous vaginal delivery at 40 weeks of gestational age. The patient had a normal blood pressure during pregnancy, indicating that she did not have preeclampsia. She had a history of normal uncomplicated vaginal delivery at 21 and 25 years of age; this was her third pregnancy. She had not undergone epidural anaesthesia or been given any vasoactive drugs. Although the patient's headache improved the day after disease onset, it re-emerged 17 days after onset, and again improved 18 days after onset. She was referred to our hospital 19 days after disease onset. On examination, her blood pressure was 112/71 mmHg. Neurological examinations revealed no abnormalities. Brain diffusion-weighted imaging showed an intensified signal in the splenium of the corpus callosum (Figure A). The apparent diffusion coefficient (ADC) indicated a low signal in the same area (Figure B). MRA showed segmental constriction of the left middle cerebral artery and bilateral posterior cerebral arteries (Figure D). A blood analysis showed no serological evidence of collagen disease. A cerebrospinal fluid analysis revealed an opening pressure of $100 \mathrm{~mm} \mathrm{H} \mathrm{H}_{2} \mathrm{O}$, no red blood cells, 1 white blood cell $/ \mathrm{mm}^{3}$, a glucose level of $62 \mathrm{mg} / \mathrm{dL}$ and a protein level of $17 \mathrm{mg} / \mathrm{dL}$. A differential diagnosis of eclampsia was excluded based on the clinical findings. Her symptoms did not worsen or relapse, despite not using either calcium-channel blockers or any other agents. MRI and MRA 20 days after the first ex-

${ }^{1}$ Department of Neurology, Chiba Rosai Hospital, Japan and ${ }^{2}$ Department of Neurology, Graduate School of Medicine, Chiba University, Japan Received for publication January 26, 2016; Accepted for publication March 7, 2016

Correspondence to Dr. Akiyuki Hiraga, hiragaa@yahoo.co.jp 


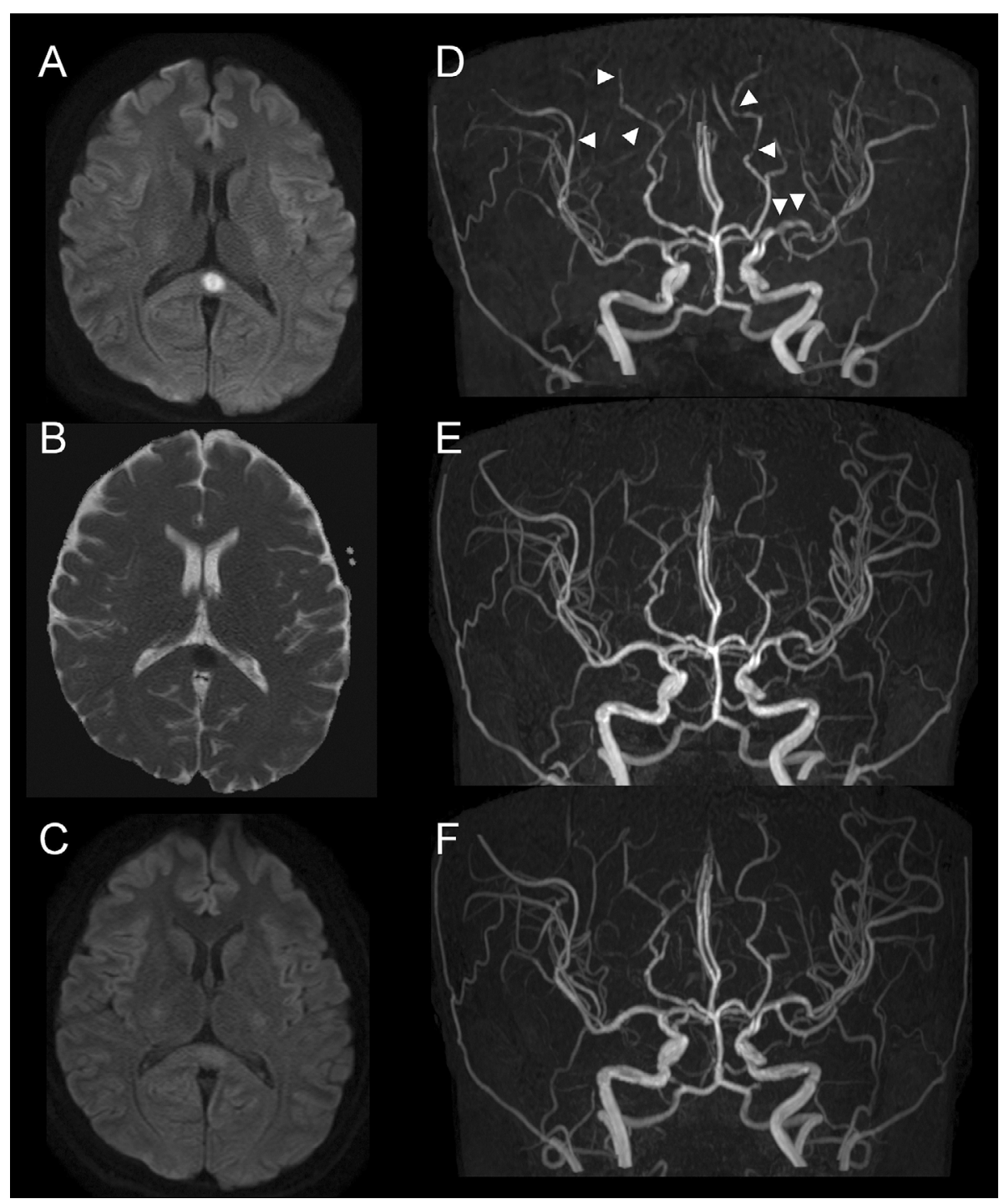

Figure. (A) Brain axial diffusion-weighted images (DWI) at first examination (19 days after onset) showing signal hyperintensity in the splenium of the corpus callosum. (B) The apparent diffusion coefficient indicating a low signal in the same area. (C) DWI at 9 days after the first examination showing splenial lesion recovery. (D-F) Serial magnetic resonance angiography showing focal constriction of the bilateral posterior cerebral arteries and the middle cerebral arteries (arrow head) and serial recovery $(D$, at first examination; $E, 9$ days after the first examination; and $F, 20$ days after the first examination).

amination were normal with a complete resolution of the TSL and all other vascular abnormalities (Figure C and F). A clinical diagnosis of RCVS with a TSL was made.

\section{Discussion}

Based on the typical and main symptoms of RCVS, our patient experienced a thunderclap headache immediately after delivery. The MRA abnormalities of RCVS and MRI abnormalities of TSL with serial recovery in our patient indicated an association between delivery and the development of both RCVS and TSL. RCVS-associated MRI abnormalities include three types of stroke (cerebral infarction, convexity subarachnoid haemorrhage, and intracerebral haemorrhage) and reversible brain edema such as PRES (1). In our case, the decreased ADC indicated cytotoxic oedema clearly distinct from PRES, as the ADC levels do not decrease in PRES.

Only two cases of RCVS with TSLs have been reported to date, and both cases, like the present case, occurred postpartum (Table) $(2,3)$. Although the timing and clinical features, such as signs, symptoms and serial MRI findings varied in these three cases, they indicated that delivery may be strongly associated with the development of both TSLs and RCVS. The important difference between the previously reported two cases and our case was the presence of neurological symptoms/sings. Our patient did not develop either seizures or neurological deficits and also had a normal blood pressure. Postpartum/peripartum patients with headaches, seizure, dysarthria, or eclampsia are immediately suspected of having neuroimaging abnormalities and require an urgent diagnostic work-up to identify the cause. Meanwhile, RCVS 
Table. Previously Reported and Present Case(s) of Reversible Cerebral Vasoconstriction Syndrome with Transient Splenial Lesion.

\begin{tabular}{|c|c|c|c|c|c|c|c|c|c|}
\hline Age/sex & Symptoms & Delivery & $\begin{array}{l}\text { Onset } \\
\text { from delivery }\end{array}$ & Eclampsia & $1^{\text {st }}$ MRI & $2^{\text {nd }} M R I$ & $3^{\text {rd }}$ MRI & Prognosis & References \\
\hline $34 \mathrm{~F}$ & Headache, GTC & Vaginal & Immediate & Yes & PRES (at onset) & SV (2 weeks) & TSL (1 month) * & Good & $2012(2)$ \\
\hline $33 \mathrm{~F}$ & Headache, SD & Caesarean & day 17 & No & SV with TSL (at onset) & Normal (day 28) & ND & Good & $2014(3)$ \\
\hline $28 \mathrm{~F}$ & Headache & Vaginal & Immediate & No & SV with TSL (day 20) & Mild SV (day 29) & Normal (day 40) & Good & Present case \\
\hline
\end{tabular}

GTC: generalized tonic-clonic seizure, SD: speech disturbance, PRES: posterior reversible encephalopathy syndrome, SV: segmental vasoconstriction demonstrated by MRA, TSL: transient splenial lesion, ND: not described, MRI: magnetic resonance imaging. $* 4^{\text {th }}$ MRI (performed 2 months after onset and showed no abnormalities).

cases presenting with headache only, like in our case, might be overlooked or underestimated. Postpartum RCVS is a known albeit rare condition (4). Postpartum TSLs are also rare; however, a few cases of TSLs (without RCVS) after pregnancy have been reported (5-8). TSLs are associated with a variety of disorders, including infection, antiepileptic drug withdrawal, high-altitude cerebral oedema, metabolic disturbance and others (9). Despite the large number of aetiologies, it remains uncertain as to why TSLs selectively occur in the splenium; moreover, the exact pathogenesis of TSLs remains elusive. Most TSL cases show data supporting cytotoxic oedema (9). The splenium differs from other parts of the corpus callosum with respect to its vascular supply; the rostrum, genu and trunk, which constitute the anterior part of the corpus callosum, are supplied by branches of the artery originating from the anterior circulation, whereas the splenium is supplied by both the anterior and posterior branches of the artery (10). However, because of the reversibility of the lesions and the absence of any other lesions in vascular distributions, it is unlikely that TSLs are the result of ischaemia (11). It has been postulated that the splenium has a specific vulnerability to excitotoxic injury in metabolic diseases, making it selectively involved in differential pathological events (12). Another possible explanation for such TSL development is that it may be related to a relative 'lack of adrenergic tone', making it susceptible to hypoxic vasodilation and autoregulation failure with resultant hyperperfusion $(9,13)$. On the other hand, transient 'failure of regulation of cerebral arterial tone' with sympathetic hyperactivity seems to play a role in RCVS development (1). Although hormonal and metabolic changes during the peripartum period can contribute to the pathogenesis, of RCVS and TSL, a transient failure of regulation of arterial tone after delivery might be the common mechanism underlying both TSL and RCVS development.

Patients with TSLs are often asymptomatic, and these lesions are incidentally noticed during MRIs performed for other reasons. Moreover, RCVS presenting only with a headache is also common, and a diagnosis of RCVS depends on the MRA findings. The actual incidence of RCVS with TSLs or postpartum TSLs is therefore unknown; RCVS with TSLs may be overlooked and might therefore be a more common condition than is currently thought.
The authors state that they have no Conflict of Interest (COI).

\section{References}

1. Ducros A. Reversible cerebral vasoconstriction syndrome. Lancet Neurol 11: 906-917, 2012

2. Sekine T, Ikeda K, Hirayama T, Suzuki A, Iwasaki Y. Transient splenial lesion after recovery of cerebral vasoconstriction and posterior reversible encephalopathy syndrome: a case report of eclampsia. Intern Med 51: 1407-1411, 2012.

3. Takahashi Y, Hashimoto N, Tokoroyama H, et al. Reversible splenial lesion in postpartum cerebral angiopathy: a case report. J Neuroimaging 24: 292-294, 2014.

4. Skeik N, Porten BR, Kadkhodayan Y, McDonald W, Lahham F. Postpartum reversible cerebral vasoconstriction syndrome: review and analysis of the current data. Vasc Med 20: 256-265, 2015.

5. Maeda M, Tsukahara H, Terada $\mathrm{H}$, et al. Reversible splenial lesion with restricted diffusion in a wide spectrum of diseases and conditions. J Neuroradiol 33: 229-236, 2006.

6. Chen Z, Xu M, Shang D, Luo B. A case of reversible splenial lesions in late postpartum preeclampsia. Intern Med 51: 787-790, 2012.

7. Curtis R, Winder T, Scott J, Hill MD. Benign post-partum reversible restricted diffusion lesion of the splenium. Can J Neurol Sci 40: 89-90, 2013.

8. Udaya SC, Chauhan BN, Philip VJ. Bright splenium of a psychotic mind. Ann Indian Acad Neurol 18: 80-83, 2015.

9. Garcia-Monco JC, Cortina IE, Ferreira E, et al. Reversible splenial lesion syndrome (RESLES): what's in a name? J Neuroimaging 21: e1-e14, 2011.

10. Kahilogullari G, Comert A, Ozdemir M, et al. Arterial vascularization patterns of the splenium: an anatomical study. Clin Anat 26: 675-681, 2013.

11. Tada H, Takanashi J, Barkovich AJ, et al. Clinically mild encephalitis/encephalopathy with a reversible splenial lesion. Neurology 63: 1854-1858, 2004.

12. Zhang S, Ma Y, Feng J. Clinicoradiological spectrum of reversible splenial lesion syndrome (RESLES) in adults: a retrospective study of a rare entity. Medicine (Baltimore) 94: e512, 2015.

13. Hackett PH, Yarnell PR, Hill R, Reynard K, Heit J, McCormick J. High-altitude cerebral edema evaluated with magnetic resonance imaging: clinical correlation and pathophysiology. JAMA 280: 1920-1925, 1998.

The Internal Medicine is an Open Access article distributed under the Creative Commons Attribution-NonCommercial-NoDerivatives 4.0 International License. To view the details of this license, please visit (https://creativecommons.org/licenses/ by-nc-nd/4.0/).

(C) 2016 The Japanese Society of Internal Medicine

http://www.naika.or.jp/imonline/index.html 